\title{
Molecular characterization of a novel secretory phospholipase A2 as coding gene from venom glands of Iranian Scorpio maurus (Arachnida: Scorpionidae)
}

\section{Parisa Soltan-Alinejad}

Shiraz University of Medical Sciences

Hamzeh Alipour

Shiraz University of Medical Sciences

Aboozar Soltani

Shiraz University of Medical Sciences

Qasem Asgari

Shiraz University of Medical Sciences

Amin Ramezani

Shiraz University of Medical Sciences

Davood Mehrabani

University of Alberta, Canada

Kourosh Azizi ( $\nabla$ azizik@sums.ac.ir)

Shiraz University of Medical Sciences https://orcid.org/0000-0001-7051-2536

Research article

Keywords: Scorpio maurus, Phospholipase A2, PLA2, Scorpion, Iran

Posted Date: May 24th, 2021

DOI: https://doi.org/10.21203/rs.3.rs-549530/v1

License: (c) (1) This work is licensed under a Creative Commons Attribution 4.0 International License. Read Full License 


\section{Abstract \\ Background}

Scorpions as a venomous arthropod can defend and capture the prey by secretion of venom into their venomic sacs. The venom is a mixture of a variety of compounds with specific biological activities, such as phospholipase A2 (PLA2) enzyme to be present in scorpion venom. This study is the first investigation of structural and molecular characterization of PLA2 from Iranian scorpion, $S$. maurus that can be added to the literature when targeting molecular characterization of PLA2 coding gene from venom glands of Iranian S. maurus.

\section{Results}

S. maurus PLA2 with 432 base pair (bp) nucleotide length encoded a protein of 144 amino acid residues and 16.34 kilodaltons $(\mathrm{kDa})$. According to DiANNA 1.1 web server prediction, this protein had 5 disulfide bridges. The deposited PLA2 in GenBank (MW241004) was very similar to S. maurus (MF347455.1) with $95.14 \%$ identity. The phylogenetic analysis indicated that our PLA2 had a close relationship with insect PLA2. Our PLA2 had $38.83 \%$ identity with bee venom PLA2 according to SWISS-MODEL prediction and $100 \%$ confidence and $39 \%$ identity with insect phospholipase A2 family, which was predicted by Phyre2. Our PLA2 was similar to chain A of bee venom PLA2. Conserved domains on target PLA2 contained 17118 amino acid in length and 45-52 amino acids in the active site. There was no signal peptide.

\section{Conclusions}

In the light of our results, the coding sequence of characterized PLA2 was a member of the PLA2 superfamily. According to the three-dimensional structure prediction, the target PLA2 with five disulfide bonds has a very high similarity to the structure of phospholipase A2 belonged to the group III subfamily. Interestingly, the active site residue of target PLA2 also has the His-Asp acid pair and the effective amino acid in catalytic domain of it wasHis ${ }^{49}$.

\section{Background}

The order of scorpions is the oldest one of venomous arthropods that have been living over 400 million years ago [1, 2]. So far, over 2,360 scorpion species have been identified and classified as 19 families [3]. The scorpion species have venom glands used to secrete venom into venom sacs to defend and capture the prey [4]. The secreted compounds from the scorpion venom gland are a complex mixture of proteins, non-proteins, and some unknown compounds with unknown function including inorganic salts, mucoproteins, carbohydrates, peptides, lipids, free amino acids, nucleotides, water, and enzymes such as phospholipase A2 (PLA2) [5-7]. 
PLA2 is an enzyme that hydrolyzes glycerophospholipids at position of sn-2 of phospholipids and releases free fatty acids [oleic acid (OA) and arachidonic acid (AA)] and lysophospholipids [8, 9]. This superfamily is classified into 15 groups and many subgroups [10]. PLA2 superfamily has six principal types including cytosolic PLA2 (cPLA2), platelet-activating factor cetyl hydrolases (PAF-AH), $\mathrm{Ca}^{2+}$ independent PLA2s (iPLA2s), lysosomal PLA2s, adipose specific PLA (AdPLA $\left._{2}\right)$ and secreted phospholipase A2 (sPLA2) [10-12].

SPLA2 as calcium-depend enzyme, is a typical enzyme in the venom of snake, lizard, bee, and scorpion that has been identified till now $[8,13-15]$. This low-molecular-weight enzyme is $13-15$ kilodaltons $(\mathrm{kDa})$ [16]. So far, 17 isoforms of SPLA2 have been identified and divided in groups I-III, V, IX-XIV [17]. sPLA2 from the venom glands of scorpions is classified as group III subfamily [18]. sPLA2 has been identified from different species of scorpions till now, including Pandinus imperator [19, 20], Hemiscorpius lepturus [21, 22], Anuroctonus phaiodactylus [18], Heterometrus fulvipes [23], Heterometrus laoticus [8] and Scorpio maurus [24].

Iran as a country located in the Middle East, due to its climate, has a good potential for scorpions living [25]. Fars province which is located in the southwest of Iran has hot and humid weather and scorpions are important public health problem in this region [26]. Scorpio maurus as a species of Scorpionidae family is a considerable species in this province [27]. The aim of this study was to identify PLA2 from the venom glands of Iranian scorpion, $S$. maurus based on a molecular characterization.

\section{Results}

\section{Characterization of the PLA2 coding gene}

To decide the PLA2 coding sequence, polymerase chain reaction (PCR) reaction, which was performed by specific primers on the synthesized complementary DNA (CDNA), appeared as a fragment close to the awaited size of 432 base pair (bp) (Figure1). The PLA2 sequence was deposited in GenBank under accession number (MW241004).

\section{Analysis of cDNA and protein sequence of $S$. maurus PLA2}

S. maurus PLA2 protein contained a 432 bp open reading frame (ORF) that encoded a protein of 144 amino acid residues with a predicted molecular mass of $16.34 \mathrm{kDa}$ (Figure 2). The amino acid sequence of concluding PLA2 was evaluated by GenRunner software. The BLAST result showed that the protein sequence was very similar to S. maurus PLA2 (GeneBank: MF347455.1) with 95.14\% identity. Other listed proteins with high identity levels in BLAST search were $74.07 \%$ to Hemilipin from $H$. lepturus (GeneBanke: KX924472.1), 78.12\% to PLA2 from O. cayaporum (GeneBanke: FM998793.1), and 74.12\% to PLA2 from A. phaiodactylus (GeneBanke: EF364043.1). To ensure, S. maurus PLA2, protein sequence was assessed using protein BLAST alignment. At first glance, all proteins revealed association with PLA2 enzyme.

\section{Phylogenetic analysis}


Initial tree(s) for the heuristic search were received automatically by applying Neighbor-Join and BioNJ algorithms to a matrix of pairwise distances estimated using the Maximum Composite Likelihood (MCL) approach, and then selecting the topology with higher-level logarithm likelihood value. Instructive branch lengths were typically drawn to scale and showed the number of substitutions per site $(0.2)$. The significant relationship between insect PLA2 was quite obvious. In addition, the phylogenetic analyses showed that the enzyme detected in human differed significantly from that of insects (Figure 3).

\section{Structural characteristics of S. maurus PLA2}

The consequences indicated that all selected proteins were associated with insect PLA2 .Above all, high similar to S. maurus PLA2 (GeneBank: AVD99009.1) with $90.28 \%$ identity. Sequence alignment of target S. maurus with three other species of scorpions including S. maurus (AVD99009.1), H. fulvipes (Q3YAU5.1), and H. lepturus (A0A1L4BJ46.1) were performed by Clustal Omega and conserved amino acids were highlighted (Figure 2). The conserved amino acids between the two species sequences of target $S$. maurus and S. maurus (AVD99009.1) were analyzed. The target $S$. maurus PLA2 did not have similarity with S. maurus PLA2 (AVD99009.1) in 13 amino acids. The amino acid compounds of S. maurus PLA2 were shown in Table 1. The predicted disulfide bonds in five positions were between (Cys8 and Cys46), (Cys25 and Cys46), (Cys45 and Cys46), (Cys52 and Cys75), and (Cys77 and Cys84) (Figure 4).

\section{Three-Dimensional structure prediction}

The three dimensional (3D) structure of $S$. maurus PLA2 by SWISS-MODEL demonstrated that the target PLA2 was similar to chain A of bee venom PLA2, group III subfamily, with $38.83 \%$ identity. The predicted 3D structure demonstrated that target PLA2 of $S$. maurus was similar to chain A of bee venom PLA2. The 3D structure of $S$. maurus PLA2 by Phyre2 displayed that the target sequence close to insect PLA2 family with $100 \%$ confidence and $39 \%$ identity. On the other hand, The S. maurus PLA2 was similar to vertebrate PLA2 with $97.3 \%$ confidence and $33 \%$ identity.

\section{Active site structure}

There was no signal peptide in target PLA2. The comparison of our PLA2 sequence with similarly characterized related proteins displayed a conserved catalytic site (active site), which was common in PLA2 superfamily of secretory and cytosolic enzymes. Conserved domains on target PLA2 contained 17118 amino acids length (Figure 5). According to ExPASy-PROSITE online tool, the active site of target PLA2 contained 45-52 amino acids length which was conserved among different species of scorpions (Figure 2). The catalytic domain included "CCRTHDXC motif," which was the binding domain that supported the active site in PLA2 superfamily.

\section{Prediction of protease cleavage sites}

Based on PROSPER, three types of protease families cleavage the target PLA2 sequence were determined and shown in Table 3. According to PeptideCutter web server, the prediction enzymes including Caspase-1 
to -10, Enterokinase, Factor Xa, GranzymeB, and Thrombin were demonstrated not to cut the target PLA2.

\section{Discussion And Conclusion}

The current study was the first investigation of structural and molecular characterization of PLA2 from venom glands of Iranian $S$. maurus that can be added to the literature when targeting molecular characterization of PLA2 coding gene from venom glands of Iranian S. maurus. Phospholipase A2 (PLA2) is functionaries of the inflammatory process which catalyzes the hydrolyze phospholipids at the sn-2 position of the glycerol backbone and releases fatty acid and lysophospholipids [28, 29]. Group III subfamily of PLA2s have been identified from various sources such as reptiles [30, 31], mammals [32], parasites [33], and arthropods including scorpion [18, 21]. The coding sequence of PLA2 was detected from $S$. maurus venom glands for the first time in Iran. The detected PLA2 has encoded a protein of 144 amino acid residue to be $16.34 \mathrm{kDa}$. Till now, several studies reported the genes encoding phospholipases A2 from different species of scorpions including Hemilipin from $H$. lepturus [21], Imperatoxin I and Phospholipin from P. imperator [19, 20], Phaiodactylipin from the A. phaiodactylus [18], Heteromtoxin from Heterometrus laoticus [8], PhospholipaseA2 from H. fulvipes [34], MtPLA2 from Mesobuthus tumulus [35], Sm-PLVG from Tunesian S. maurus [24], and PhospholipaseA2 from Hadrurus gertschi [36].

The results of Blast in NCBI showed that the detected sequence from the Iranian scorpio maurus has a very high similarity to the same sequence of Tunesian S. maurus (GeneBank: MF347455.1) [37], while this level of similarity is very low compared to other species of scorpions. Comparing the coding sequence of target PLA2 with other characterized PLA2 from different species of scorpions, was shown that our PLA2 was a member of the PLA2 superfamily.

Phylogenetically, there is a distant relationship between group I, II, and III of phospholipase A2, but at the site of calcium-binding and the region of active site, they are quite similar [38, 39]. In the current study, the result of likelihood analysis showed that the characterized PLA2 from Iranian S. maurus and other arthropods, scorpions and Apis mellifera (FE373554.1), are well clustered, whereas this one from Homo sapience (M86400.1) is distantly located. similar to the phylogenetic relationship of phospholipases A2 from A. phaiodactylus [39].

The difference in 13 amino acids residue in the gene encoding sequence of phospholipase A2 enzyme between the Iranian and Tunisian S. maurus is most likely due to differences in the geographical condition in which they live. The residues $\mathrm{Asp}^{2}, \mathrm{Leu}^{9}, \mathrm{Phe}^{17}, \mathrm{Glu}^{30}, \mathrm{Glu}^{32}, \mathrm{Ser}^{67}, \mathrm{Pro}^{69}, \mathrm{Met}^{86}$, $\mathrm{Asp}^{90}, \mathrm{Thr}^{95}, \mathrm{Asn}^{102}, \mathrm{Lys}^{109}, \mathrm{Tyr}^{110}$ of target $S$. maurus PLA2 were Val, Ser, Leu, Val, Lys, Phe, Phe, Thr, Asn, Asp, Asp, Asp, Asn in Tunisian S. maurus (AVD99009.1), respectively. Certainly, the difference in the amino acid residue is greater among different species of scorpions.

Based on the results of the three dimensional structure prediction, the phospholipaseA2 encoding gene isolated from the Iranian $S$. maurus has a very high similarity to the structure of phospholipase A2 
belonged to group III subfamily, but similarity to vertebrates' phospholipase A2 was smaller. Phylogenetic analysis also confirms these results. Similar to characterized PLA2 of A. phaiodactylus [39], Imperatoxin I of $P$. imperator [19], our PLA2 is closely related to genomic structure of $A$. mellifera, the only known representative structure in group III PLA2. Evaluation of the 3D structure of the target gene revealed that the identified protein was very similar to the chain A of characterized PLA2 from bee venom. Four disulfide bonds were predicted for phospholipase A2 of $M$. tamulus at the position of eight cysteines (Cys8-Cys30), (Cys29-Cys68), (Cys35-Cys61) and (Cys59-Cys96) [35]. It is similar to the position of the human group III PLA2 disulfide bonds in ten cysteines (Cys8-Cys30), (Cys29-Cys68), (Cys35-Cys61), (Cys59-Cys91), and (Cys101-Cys113) [40], while different positions were predicted to the five disulfide bonds in target PLA2. The histidine-aspartic (His-Asp) acid pair has been suggested to be necessary for the catalytic mechanism of the phospholipase A2 [41]. In this study, the active site residue of target PLA2 also has the His-Asp acid pair and the effective amino acid in catalytic domain of target PLA2 was $\mathrm{His}^{49}$, while His ${ }^{34}$ played a major role in the active site residue of bee's venom and Tunisian S. maurus [37].

\section{Methods}

\section{Scorpion collection}

The scorpions were collected from Fars Province, Zarrin Dasht County, south-west of Iran, and transferred alive to the laboratory of Medical Entomology in Shiraz University of Medical Sciences, Shiraz, Iran. Samples were identified via a valid key [42]. Before RNA extraction, venom of the collected scorpions was milked manually to release the venom. Three days after venom milking, the telson of the scorpions were separated and stored at $-70 \circ \mathrm{C}$. Other parts of the body were stored at ethanol $96 \%$ and were kept in the archives of the Museum of the department of Medical Entomology in the Shiraz University of Medical Sciences.

\section{Primer design}

To design the gene-specific primer (GSPs), the mRNA sequences of PLA2 from different species of scorpions such as S. maurus (GeneBanke: MF347455), H. leptorus (GeneBanke: KX924472), A. phaiodactylus (GeneBanke: AY571967.1), and Opisthacanthus cayaporum (GeneBanke: FM998793.1) were obtained from national center for biotechnology information (NCBI). The normal size bound was $432 \mathrm{bp}$. The specific primers were 5'-TCCAAAGAAGAAATGGA (forward primer) and 5'GTCTTTGTAGCTCTTTTTCCAGG (reverse primer).

\section{Preparation of cDNA}

Total RNA was extracted from the venom glands of $S$. maurus telson by High Pure RNA Isolation Kit, Roche ${ }^{\circledR}$. RNA sample was treated enzymatically by DNase based on manufacturer's manual. Extracted RNA concentration was measured using a Nanodrop (analytikjena ${ }^{\circledR}$ ). 


\section{Reverse Transcriptase Polymerase Chain Reaction (RT-PCR)}

According to manufacturer's instruction, $3 \mu \mathrm{L}$ of total RNA was used as a template for cDNA synthesis by AccuPower ${ }^{\circledR}$ CycleScript RT Premix with (d) (Bioneer Company, Korea). 0.1 to $1 \mu \mathrm{g}$ of RNA template was filled up to the $20 \mu \mathrm{L}$ volume with Diethylpyrocarbonate-treated water (DEPC DW) and was dissolved by vortexing. cDNA synthesis reaction was performed in four steps according to manufacturer's manual including $30 \mathrm{sec}$ at $25^{\circ} \mathrm{C}$ for primer annealing, 4 min at $45^{\circ} \mathrm{C}$ for cDNA synthesis, $30 \mathrm{sec}$ at $55^{\circ} \mathrm{C}$ for melting secondary structure and $\mathrm{CDNA}$ synthesis, and $5 \mathrm{~min}$ at $95^{\circ} \mathrm{C}$ for heat inactivation. The synthesized $\mathrm{CDNA}$ was kept at $-70^{\circ} \mathrm{C}$ for PCR amplification steps as a template.

\section{Polymerase Chain Reaction (PCR)}

The desired DNA sequences were amplified in total $20 \mu \mathrm{L}$ volume containing $10 \mu \mathrm{L}$ Taq DNA Polymerase Master Mix RED (2X), $1 \mu \mathrm{L}$ forward primer, $1 \mu \mathrm{L}$ of reverse and finally $1 \mu \mathrm{L}$ of synthesized cDNA. It was performed to 35 cycles of $30 \mathrm{~s}$ at $94^{\circ} \mathrm{C}$ as denaturation, $30 \mathrm{sec}$ at $50^{\circ} \mathrm{C}$ as annealing temperature, $30 \mathrm{sec}$ at $72^{\circ} \mathrm{C}$ as extension and $10 \mathrm{~min}$ at $72^{\circ} \mathrm{C}$ for a final extension.

\section{DNA gel purification and sequencing}

PCR products were run onto $2 \%$ tris borate EDTA (TBE) agarose gel with appropriate DNA ladder. Our specific band was observed in the gel documentation instrument. Selected band was purified according to protocol of Gene All kit. Expected size bands were sequenced using GSPs forward and reverse.

\section{Phylogenetic analysis}

To evaluate the target PLA2 protein, a phylogenetic tree was created based on different characterized related protein sequences including scorpion species, Apis mellifera, and Homo sapiens by utilizing the maximum likelihood method. Multiple sequences alignment for evolutionary analyses were conducted in MEGA7 software based on the Clustal W method [43]. The percentage of replicate trees in which the associated taxa clustered together in the bootstrap test (1000 replicates) were shown next to the branches [44].

\section{Structural characteristics of $S$. maurus PLA2}

MEGA software (Version 7.0) was used for alignments. All primers were designed by the GeneRunner (Version 4) and Oligo 7 software. The designed primers specificity was determined using Primer-BLAST on NCBI (http://blast.ncbi.nlm.nih.gov/Blast.cgi). To identify the structural features of the protein coded by PLA2 and guarantee that obtained sequence could be a part of phospholipase family proteins, the $S$. maurus PLA2 was translated by GeneRunner software (Version 4.0), and the concluded sequence was evaluated by diverse instruments. Protein BLAST was performed to decide proteins with great closeness to recognize and record their characterization. To compare and classify protein structure, similar proteins were chosen for alignment with Clustal Omaga (https://www.ebi.ac.uk/Tools/msa/clustalo/). Amino acid compounds of S.maurus PLA2 were analyzed by utilizing ProtParam online tool 
(https://web.expasy.org/protparam/). The PLA2 coding gene was translated by GeneRunner software (version 4). A protein BLAST was also carried out for more comparative relation. To disulfide bridge prediction of the target protein, DiANNA 1.1 web server was used $[45,46]$. Disulfide bridges formation are essential for biological activity in many proteins [47]. To predict disulfide bonds, DiANNA 1.1 web server (http://clavius.bc.edu/ clotelab/DiANNA/) was used.

\section{Three-Dimensional structure prediction}

In order to predict the 3D structure, the SWISS-MODEL online tool (https://swissmodel.expasy.org/) and Phyre2 (http://www.sbg.bio.ic.ac.uk/phyre2/html/page.cgi?id=index ) were used, which predicted the 3D structure of a query protein through the sequence alignment of template proteins. This model was chosen for superimposition. The predicted 3D structure was evaluated by UCSF Chimera software (Version 1.14).

\section{Active site structure}

Several online tools were used to evaluate the signal peptide of the gene in question. PLA2 breaks the sn2 position of the glycerol backbone of phospholipids, mainly in a metal-dependent reaction, to produce lysophospholipid (LysoPL) and a free fatty acid (FA) [8, 9]. Superimposition of active site was carried out by UCSF Chimera (Version 1.14) and DeepView/Swiss-pdbviewer (Version 4.10) software (Figure 3) (34). The root-mean-square deviation (RMSD) of bee venom PLA2 and target protein active site residue was calculated by Chimera software (Version 1.14) to measure the average distance between corresponding atoms in two protein chains based on carbon alpha atoms (Table 2). Active-site residues were universally conserved within protein families, displaying their key role for substrate catalysis [48]. The active site prediction was undertaken by ExPASy-PROSITE (https://prosite.expasy.org/). Several online devices were used for signal peptide analysis such as SignalP 4.1 Server (http://www.cbs.dtu.dk/services/SignalP/), Signal Signal-3L 2.0, and BLAST (http://sigpep.services.came.sbg.ac.at/signalblast.html).

\section{Prediction of protease cleavage sites}

PROSPER (protease specificity prediction Server), (https://prosper.erc.monash.edu.au/webserver.html) and PeptideCutter (http://web.expasy.org/peptide_cutter/) servers were used for prediction of the activated form of target protein. ProtParam (https://web.expasy.org/protparam/) was used for amino acid composition.

\section{Abbreviations}

PLA2: Phospholipase A2; cPLA2: cytosolic PLA2; PAF-AH: platelet-activating factor cetyl hydrolases; iPLA2s independent PLA2s; AdPLA2: adipose specific PLA2; sPLA2: secreted phospholipase A2; OA: Oleic acid; AA: Arachidonic acid; cDNA: complementary DNA; PCR: polymerase chain reaction; bp: Base pair; kDa: Kilodaltons ; ORF: open reading frame; MCL: Maximum Composite Likelihood; 3D: three dimensional structure; NCBI: national center for biotechnology information; RT-PCR: Reverse Transcriptase Polymerase Chain Reaction; $\mu L$ : Microliter; Met: Methionine; TBE: Tris borate EDTA; GSPs: Gene-Specific Primers; 
RMSD: root-mean-square deviation; Thr: Threonine; Asn: Asparagine; Lys: Lysine; Tyr: Tyrosine; Asp: Aspartic acid; Cys: Cysteines; His: Histidine; Val: Valine; Leu: Leucine; Phe: Phenylalanine; Glu: Glutamic acid; Ser: Serine; Pro: Proline.

\section{Declarations}

\section{Ethics approval and consent to participate}

No human or animal data or tissue was used in this study. But all experiments were performed in accordance with the ethical standards of the Shiraz University of Medical Sciences ethics committee (ethics committee code: IR. SUMS. REC.1399.87). Specimens (Scorpions) were not client-owned animal and we collected them from the natural habitats (Zarrin Dasht county, Fars province, South of Iran) with the permission of the Shiraz University of Medical Sciences and in coordination with the Fars Environmental Protection Organization.

\section{Consent for publication}

Not applicable

\section{Availability of data and materials}

All data generated or analyzed during this study are available upon reasonable request to the corresponding author.

\section{Competing interests}

None declared.

\section{Funding}

This investigation was funded by Shiraz University of Medical Sciences (SUMS), Shiraz, Iran (Grant No: 98-01-04-20786).

\section{Authors' contributions}

PS-A and KA conceived and designed the study and contributed to the discussion of results, HA, AS, QA and AR conceived the study and analyzed data, PS-A, KA and DM drafted the manuscript. All authors have read and approved the final manuscript.

\section{Acknowledgments}

The authors are appreciative of the financial support provided by the Shiraz University of Medical Sciences. We are very grateful to Mr. Ali Dianat for collecting scorpions. We are grateful to Mrs. Sorna Dabbaghmanesh and Mr. Amin Hosseinpour, staff of the Medical Entomology Laboratory of School of Health for their kind support. This article is part of the results of the first author's Ph.D. thesis in Medical 
Entomology and Vector Control at Department of Medical Entomology and Vector Control, School of Public Health, Shiraz University of Medical Sciences (SUMS), Shiraz, Iran. This investigation was funded by Shiraz University of Medical Sciences (SUMS), Shiraz, Iran (Grant No: 98-01-04-20786).

\section{References}

1. Soleglad, ME, Fet, V: High-level systematics and phylogeny of the extant scorpions (Scorpiones: Orthosterni). Euscorpius 2003, 2003(11):1-56, doi:10.18590/euscorpius.2003.vol2003.iss11.1.

2. Rokyta, DR, Ward, MJ: Venom-gland transcriptomics and venom proteomics of the black-back scorpion (Hadrurus spadix) reveal detectability challenges and an unexplored realm of animal toxin diversity. Toxicon 2017, 128:23-37, doi:https://doi.org/10.1016/j.toxicon.2017.01.014.

3. Mullen, GR, Sissom, WD: Scorpions (Scorpiones). In: Medical and Veterinary Entomology. Edited by Mullen GR, Durden LA, (Third Edition) edn: Academic Press; 2019: 489-504.

4. YiĞiT, N, BAYRAM, A, DANIŞMAN, T: Functional morphology of venom apparatus of Euscorpius mingrelicus (Scorpiones: Euscorpiidae). J Appl Biol 2007, 1(2):27-31.

5. Ding, J, Chua, P-J, Bay, B-H, Gopalakrishnakone, P: Scorpion venoms as a potential source of novel cancer therapeutic compounds. Exp Biol Med 2014, 239(4):387-393, doi:https://doi.org/10.1177/1535370213513991.

6. Salem, ML, Shoukry, NM, Teleb, WK, Abdel-Daim, MM, Abdel-Rahman, MA: In vitro and in vivo antitumor effects of the Egyptian scorpion Androctonus amoreuxi venom in an Ehrlich ascites tumor model. Springerplus 2016, 5(1):570, doi:10.1186/s40064-016-2269-3.

7. Possani, L: Structure of scorpion toxins. In: Handbook of natural toxins. 1984.

8. Incamnoi, P, Patramanon, R, Thammasirirak, S, Chaveerach, A, Uawonggul, N, Sukprasert, S et al: Heteromtoxin ( $\mathrm{HmTx}$ ), a novel heterodimeric phospholipase A2 from Heterometrus laoticus scorpion venom. Toxicon 2013, 61:62-71, doi:https://doi.org/10.1016/j.toxicon.2012.10.012.

9. Six, DA, Dennis, EA: The expanding superfamily of phospholipase A2 enzymes: classification and characterization. Biochim Biophys Acta 2000, 1488(1):1-19, doi:https://doi.org/10.1016/S13881981(00)00105-0.

10. Burke, JE, Dennis, EA: Phospholipase A2 structure/function, mechanism, and signaling. J Lipid Res 2009, 50(Supplement):S237-S242, doi:10.1194/jlr.R800033-JLR200.

11. Schaloske, RH, Dennis, EA: The phospholipase A2 superfamily and its group numbering system. Biochim Biophys Acta Gen Subj 2006, 1761(11):1246-1259, doi:10.1016/j.bbalip.2006.07.011.

12. Murakami, M, Taketomi, Y, Miki, Y, Sato, H, Hirabayashi, T, Yamamoto, K: Recent progress in phospholipase A囚 research: from cells to animals to humans. Prog Lipid Res 2011, 50(2):152-192, doi:10.1016/j.plipres.2010.12.001.

13. Doley, R, Zhou, X, Kini, RM: Snake venom phospholipase A2 enzymes. In: Handbook of venoms and toxins of reptiles. Edited by Stephen P. Mackessy SPM, vol. 1, 1st Edition edn; 2010: 173-205. 
14. Carballido, JM, Carballido-Perrig, N, Terres, G, Heusser, CH, Blaser, K: Bee venom phospholipase A2specific $T$ cell clones from human allergic and non-allergic individuals: cytokine patterns change in response to the antigen concentration. Eur J Immunol 1992, 22(6):1357-1363, doi: https://doi.org/10.1002/eji.1830220605.

15. Perumal Samy, R, Gopalakrishnakone, P, Thwin, M, Chow, T, Bow, H, Yap, E et al: Antibacterial activity of snake, scorpion and bee venoms: a comparison with purified venom phospholipase $A 2$ enzymes. J Appl Microbiol 2007, 102(3):650-659, doi:10.1111/j.1365-2672.2006.03161.x.

16. Tatulian, SA: Toward Understanding Interfacial Activation of Secretory Phospholipase A2 (PLA2): Membrane Surface Properties and Membrane-Induced Structural Changes in the Enzyme Contribute Synergistically to PLA2 Activation. Biophys J 2001, 80(2):789-800, doi:https://doi.org/10.1016/S0006-3495(01)76058-4.

17. Quach, ND, Arnold, RD, Cummings, BS: Secretory phospholipase A2 enzymes as pharmacological targets for treatment of disease. Biochem Pharmacol 2014, 90(4):338-348, doi:https://doi.org/10.1016/j.bcp.2014.05.022.

18. Valdez-Cruz, NA, Batista, CV, Possani, LD: Phaiodactylipin, a glycosylated heterodimeric phospholipase A2 from the venom of the scorpion Anuroctonus phaiodactylus. Eur J Biochem 2004, 271(8):1453-1464, doi: 10.1111/j.1432-1033.2004.04047.x.

19. Zamudio, FZ, Conde, R, Arévalo, C, Becerril, B, Martin, BM, Valdivia, HH et al: The mechanism of inhibition of ryanodine receptor channels by imperatoxin I, a heterodimeric protein from the scorpion Pandinus imperator. J Biol Chem 1997, 272(18):11886-11894, doi:10.1074/jbc.272.18.11886.

20. Conde, R, Zamudio, FZ, Becerril, B, Possani, LD: Phospholipin, a novel heterodimeric phospholipase A2 from Pandinus imperator scorpion venom. FEBS Lett 1999, 460(3):447-450, doi:https://doi.org/10.1016/S0014-5793(99)01392-7.

21. Jridi, I, Catacchio, I, Majdoub, H, Shahbazeddah, D, El Ayeb, M, Frassanito, MA et al: Hemilipin, a novel Hemiscorpius lepturus venom heterodimeric phospholipase A2, which inhibits angiogenesis in vitro and in vivo. Toxicon 2015, 105:34-44, doi:https://doi.org/10.1016/j.toxicon.2015.08.022.

22. Jridi, I, Catacchio, I, Majdoub, H, Shahbazzadeh, D, El Ayeb, M, Frassanito, MA et al: The small subunit of Hemilipin2, a new heterodimeric phospholipase A2 from Hemiscorpius lepturus scorpion venom, mediates the antiangiogenic effect of the whole protein. Toxicon 2017, 126:38-46, doi:https://doi.org/10.1016/j.toxicon.2016.12.001.

23. Ramanaiah, M, Parthasarathy, P, Venkaiah, B: Purification and properties of phospholipase A2 from the venom of scorpion,(Heterometrus fulvipes). Int J Biochem 1990, 20(5):931-940.

24. Louati, H, Krayem, N, Fendri, A, Aissa, I, Sellami, M, Bezzine, S et al: A thermoactive secreted phospholipase A2 purified from the venom glands of Scorpio maurus: Relation between the kinetic properties and the hemolytic activity. Toxicon 2013, 72:133-142, doi:https://doi.org/10.1016/j.toxicon.2013.06.017.

25. Dehghani, R, Arani, MG: Scorpion sting prevention and treatment in ancient Iran. J Tradit Med 2015, 5(2):75-80, doi:https://doi.org/10.1016/j.jtcme.2014.11.007. 
26. Alimohamadi, Y, Rajabi, A, H, A, Esmaeelzadeh, F, M, G: An Epidemiological Study on Scorpion Stings in Khuzestan Province (Haft Gel region), 2011-2012. Sadra Med Sci J 2014, 3:13-20.

27. Shahbazzadeh, D, Amirkhani, A, Djadid, ND, Bigdeli, S, Akbari, A, Ahari, $\mathrm{H}$ et al: Epidemiological and clinical survey of scorpionism in Khuzestan province, Iran (2003). Toxicon 2009, 53(4):454-459, doi:https://doi.org/10.1016/j.toxicon.2009.01.002.

28. Barahoei, H, Navidpour, S, Aliabadian, M, Siahsarvie, R, Mirshamsi, O: Scorpions of Iran (Arachnida: Scorpiones): Annotated checklist, DELTA database and identification key. $J$ insect biodivers 2020, 6(4):375-474.

29. Kumar, S, Stecher, G, Tamura, K: MEGA7: molecular evolutionary genetics analysis version 7.0 for bigger datasets. Mol Biol Evol 2016, 33(7):1870-1874, doi:10.1093/molbev/msw054.

30. Felsenstein, J: Confidence limits on phylogenies: an approach using the bootstrap. Evolution 1985, 39(4):783-791, doi:https://doi.org/10.2307/2408678.

31. Ferrè, F, Clote, P: DiANNA: a web server for disulfide connectivity prediction. Nucleic Acids Res 2005, 33(suppl_2):W230-W232, doi:10.1093/nar/gki412.

32. Ferrè, F, Clote, P: Disulfide connectivity prediction using secondary structure information and diresidue frequencies. Bioinformatics 2005, 21(10):2336-2346, doi:10.1093/bioinformatics/bti328.

33. Bulaj, G: Formation of disulfide bonds in proteins and peptides. Biotechnol Adv 2005, 23(1):87-92, doi:10.1016/j.biotechadv.2004.09.002

34. Desjardins, M, Mak, WS, O'Brien, TE, Carlin, DA, Tantillo, DJ, Siegel, JB: Systematic Functional Analysis of Active-Site Residues in I-Threonine Dehydrogenase from Thermoplasma volcanium. ACS omega 2017, 2(7):3308-3314, doi:https://doi.org/10.1021/acsomega.7b00519.

35. Tischfield, JA: A reassessment of the low molecular weight phospholipase A2 gene family in mammals. J Biol Chem 1997, 272(28):17247-17250, doi:10.1074/jbc.272.28.17247.

36. Van Deenen, L, De Haas, GH, Heemskerk, CT: Hydrolysis of synthetic mixed-acid phosphatides by phospholipase A from human pancreas. Biochim Biophys Acta 1963, 67:295-304, doi: 10.1042/bj0280476

37. Serrano, SM, Reichl, AP, Mentele, R, Auerswald, EA, Santoro, ML, Sampaio, CA et al: A novel phospholipase A2, BJ-PLA2, from the venom of the snake Bothrops jararaca: purification, primary structure analysis, and its characterization as a platelet-aggregation-inhibiting factor. Arch Biochem Biophys 1999, 367(1):26-32, doi:https://doi.org/10.1006/abbi.1999.1230.

38. Pereañez, JA, Núñez, V, Huancahuire-Vega, S, Marangoni, S, Ponce-Soto, LA: Biochemical and biological characterization of a PLA2 from crotoxin complex of Crotalus durissus cumanensis. Toxicon 2009, 53(5):534-542, doi:https://doi.org/10.1016/j.toxicon.2009.01.021.

39. Hariprasad, G, Kumar, M, Kaur, P, Singh, TP, Kumar, RP: Human group III PLA2 as a drug target: structural analysis and inhibitor binding studies. Int J Biol Macromol 2010, 47(4):496-501, doi:https://doi.org/10.1016/j.ijbiomac.2010.07.004.

40. Hariprasad, G, Kaur, P, Srinivasan, A, Singh, TP, Kumar, M: Structural analysis of secretory phospholipase A 2 from Clonorchis sinensis: therapeutic implications for hepatic fibrosis. $J$ Mol 
Model 2012, 18(7):3139-3145, doi:10.1007/s00894-011-1333-8.

41. Hariprasad, G, Hariprasad, G, Singh, B, Hariprasad, G, Singh, B, Das, U et al: Cloning, sequence analysis and homology modeling of a novel phospholipase A2 from Heterometrus fulvipes (Indian black scorpion) Full Length Research Paper. DNA Sequence 2007, 18(3):242-246, doi:https://doi.org/10.1080/10425170701243294.

42. Hariprasad, G, Kumar, M, Srinivasan, A, Kaur, P, Singh, TP, Jithesh, O: Structural analysis of a group III Glu62-phospholipase A2 from the scorpion, Mesobuthus tamulus: Targeting and reversible inhibition by native peptides. Int J Biol Macromol 2011, 48(3):423-431, doi:10.1016/j.ijbiomac.2011.01.004.

43. Schwartz, EF, Diego-Garcia, E, de la Vega, RCR, Possani, LD: Transcriptome analysis of the venom gland of the Mexican scorpion Hadrurus gertschi (Arachnida: Scorpiones). BMC Genomics 2007, 8(1):1-12, doi:10.1186/1471-2164-8-119.

44. Krayem, N, Parsiegla, G, Gaussier, H, Louati, H, Jallouli, R, Mansuelle, P et al: Functional characterization and FTIR-based 3D modeling of full length and truncated forms of Scorpio maurus venom phospholipase A2. Biochim Biophys Acta 2018, 1862(5):1247-1261, doi:https://doi.org/10.1016/j.bbagen.2018.02.004.

45. Scott, DL, Otwinowski, Z, Gelb, MH, Sigler, PB: Crystal structure of bee-venom phospholipase A2 in a complex with a transition-state analogue. Science 1990, 250(4987):1563-1566, doi:10.1126/science.2274788.

46. Valdez-Cruz, NA, Segovia, L, Corona, M, Possani, LD: Sequence analysis and phylogenetic relationship of genes encoding heterodimeric phospholipases $A 2$ from the venom of the scorpion Anuroctonus phaiodactylus. Gene 2007, 396(1):149-158, doi:https://doi.org/10.1016/j.gene.2007.03.007.

47. Hariprasad, G, Srinivasan, A, Singh, R: Structural and phylogenetic basis for the classification of group III phospholipase A 2. J Mol Model 2013, 19(9):3779-3791, doi:10.1007/s00894-013-1913-x.

48. Sekar, K, Yu, B-Z, Rogers, J, Lutton, J, Liu, X, Chen, X et al: Phospholipase A2 engineering. Structural and functional roles of the highly conserved active site residue aspartate-99. Biochemistry 1997, 36(11):3104-3114, doi:10.1021/bi961576x

\section{Tables}

Table 1. Amino acid compounds of S.maurus PLA2 were analyzed by ProtParam online tool. 


\begin{tabular}{|lll|}
\hline Amino acid type & Number & Percentage \\
\hline Glu (E) & 12 & 8.3 \\
\hline Gly (G) & 12 & 8.3 \\
\hline Lys (K) & 12 & 8.3 \\
\hline Asp (D) & 11 & 7.6 \\
\hline Ser (S) & 11 & 7.6 \\
\hline Cys (C) & 11 & 7.6 \\
\hline Thr (T) & 10 & 6.9 \\
\hline Asn (N) & 9 & 6.2 \\
\hline Ala (A) & 8 & 5.6 \\
\hline Arg (R) & 8 & 5.6 \\
\hline Leu (L) & 8 & $5.6 \%$ \\
\hline Tyr (Y) & 8 & 5.6 \\
\hline Phe (F) & 6 & 4.2 \\
\hline Pro (P) & 4 & $2.8 \%$ \\
\hline Trp (W) & 3 & 2.1 \\
\hline Val (V) & 3 & 2.1 \\
\hline Gln (Q) & 2 & 1.4 \\
\hline His (H) & 2 & 1.4 \\
\hline Ile (I) & 2 & 1.4 \\
\hline Met (M) & 2 & 1.4 \\
\hline
\end{tabular}

Table 2. The RMSD of active site residues in an important domain in Scorpio maurus and bee venom PLA2.

\begin{tabular}{|lllllllll|}
\hline Amino acid & Cys & Cys & Arg & Thr & His & Asp & His & Cys \\
\hline $\begin{array}{l}\text { Position in Scorpio maurus } \\
\text { PLA2 }\end{array}$ & 45 & 46 & 47 & 48 & 49 & 50 & 51 & 56 \\
\hline Amino acid & Cys & Cys & Arg & Thr & His & Asp & Met & Cys \\
\hline Position in bee venom PLA2 & 30 & 31 & 32 & 33 & 34 & 35 & 36 & 37 \\
\hline RMSD & 0.000 & 0.000 & 0.000 & 0.000 & 0.724 & 2.035 & 2.401 & 1.531 \\
\hline
\end{tabular}


Table 3. Predicted cleavage sites of individual proteases. Restriction site have been shown by (囚).

\begin{tabular}{|c|c|c|c|}
\hline Protease family & Proteases type & Position & Segment \\
\hline \multirow[t]{2}{*}{ Cysteine protease } & \multirow[t]{2}{*}{ Cathepsin K } & 56 & DYIG『SGET \\
\hline & & 86 & QCLM『EAYD \\
\hline \multirow[t]{4}{*}{ Metalloprotease } & \multirow[t]{4}{*}{ Matrix metallopeptidase- 9} & 63 & TKYGهLTNS \\
\hline & & 84 & FDQCØLMEA \\
\hline & & 102 & STKN囚LRNF \\
\hline & & 115 & RPQCQYVVT \\
\hline \multirow[t]{13}{*}{ Serine protease } & \multirow[t]{3}{*}{ Chymotrypsin A (cattle-type) } & 81 & EEAF囚DQCL \\
\hline & & 89 & MEAY \DKEE \\
\hline & & 138 & VATW $\triangle K K S Y$ \\
\hline & Elastase-2 & 18 & RFFIهWGGT \\
\hline & \multirow[t]{5}{*}{ Cathepsin G } & 17 & GRFF®IWGG \\
\hline & & 54 & HCDY\IGSG \\
\hline & & 62 & ETKY凶GLTN \\
\hline & & 116 & PQCY\VVTC \\
\hline & & 121 & VTCNखSNRS \\
\hline & \multirow[t]{3}{*}{ Glutamyl peptidase I } & 41 & GPLE\ADKC \\
\hline & & 79 & KCEE $\triangle A F D Q$ \\
\hline & & 132 & AGCE『NGVA \\
\hline & Thylakoidal processing peptidase & 52 & HDHC囚DYIG \\
\hline
\end{tabular}

\section{Figures}


1

2

3

4

\section{$1500 \mathrm{bp}$}

\section{$1000 \mathrm{bp}$}

$500 \mathrm{bp}$

\section{Figure 1}

Amplification of Scorpio maurus PLA2 mRNA; Lane 1 is ladder 100 bp DNA marker, Lane 2 and 3 are PLA2 (432bp), and lane 4 is negative control 
Heterometrus fulvipes

Hemiscorpius lepturus

Scorpio maurus

Scorpio maurus

Heterometrus fulvipes

Hemiscorpius lepturus

Scorpio maurus

Scorpio maurus

Heterometrus fulvipes

Hemiscorpius lepturus

Scorpio maurus

Scorpio maurus
ECRMINSMHELSRELMDSPEHTVKRASKEEMDDLVQRCSGSAEGRSWFIWPDTKWCGPGT 120 --n

KAINYTDLGYFSNLDSCCRTHDHCDNIAAGETKYGLTNEGKYTMMNCKCEATFQQCLRDV 72 DAKNESDLGPLE-ADKCCRTHDHCDYIGAGETKYGLTNKSFFTKLNCKCEAAFDQCLKES 179 EAENE SDLGPLE-ADKCCRTHDHCDYIGSGETKYGLTNSS PFTKLNCKCEEAFDQCLMEA 88 VAKNE SDLGPLE-ADKCCRTHDHCDYIGSGETKYGLTNESEFTKLNCKCEEAFDQCLTEA 88

HGPLEGKAAF---TIRKLYFGLYGNGCFNVQCPS--------------------- 103

IDRAEGSAKSSMEGLHSFYFNTYSPECYEVKCSRK---RDAECTNGIAIWKDSYKS 232

YDKEEGTAKTSTKNLRNFYFKYYRPQCYVVTCNSNRSSRDAGCENGVATWKKSYKD 144

YNKEEGDAKTSTKDLRNFYFDNYRPQCYVVTCNSKRSSRDAGCENGVATWKKSYKD 144

\section{Figure 2}

Sequence alignment of target Scorpio maurus (underlined) with three other species of scorpions including S. maurus (AVD99009.1), Heterometrus fulvipes (Q3YAU5.1), and Hemiscorpius lepturus (A0A1L4BJ46.1). Gaps are displayed by (-). Conserved amino acids among species are shaded in gray. Conserved amino acids between the same species of $\mathrm{S}$. maurus are bolded. Conserved catalytic site is assigned by $\left({ }^{*}\right)$. 

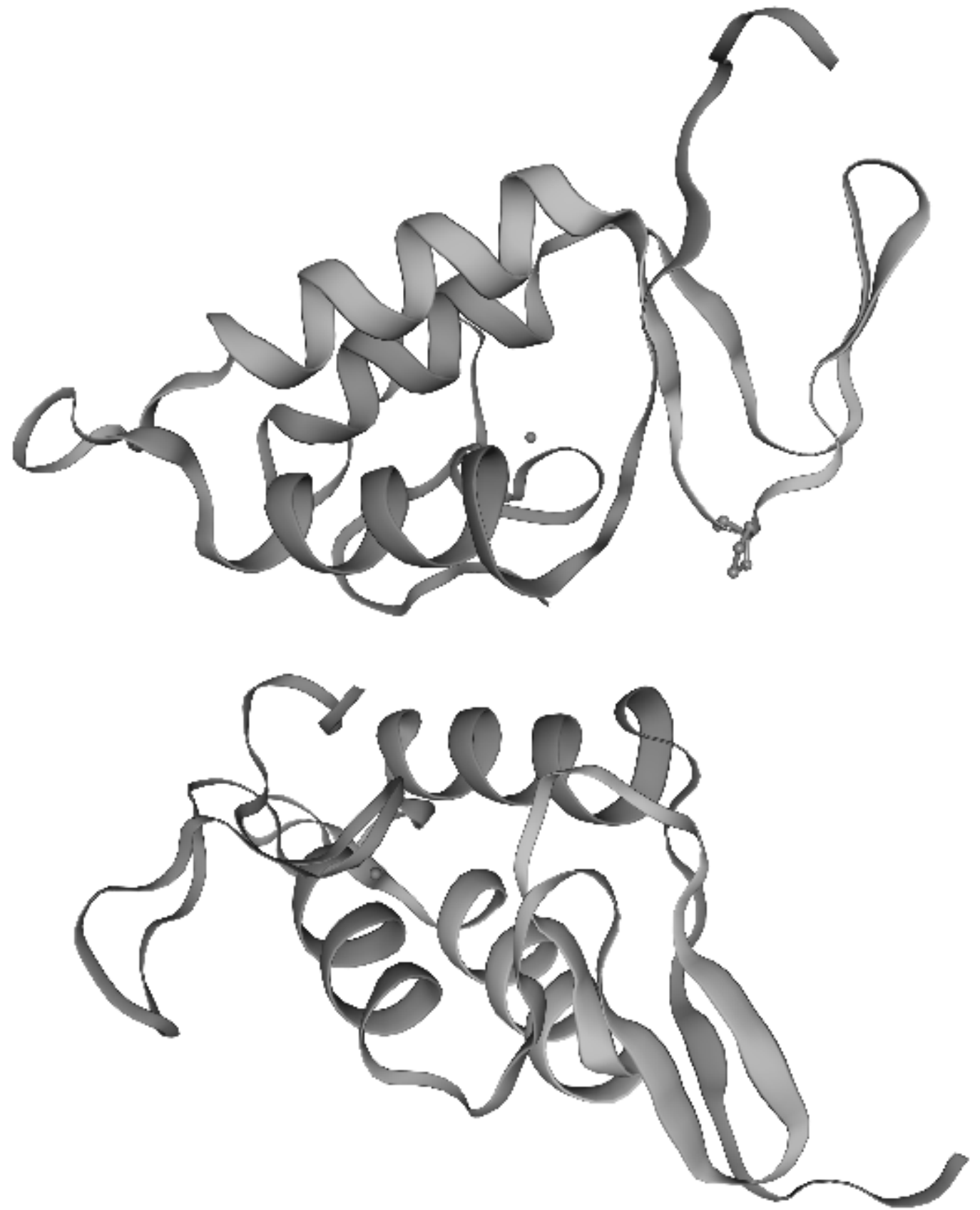

Figure 3

3D structure of PLA2 was predicted by SWISS-MODEL web server. Predicted 3D structure of homo-dimer PLA2 of S. maurus with $38.83 \%$ identity to bee venom PLA2. 


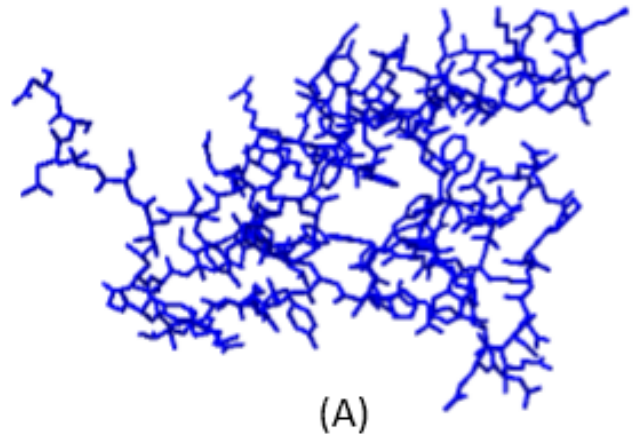

(A)

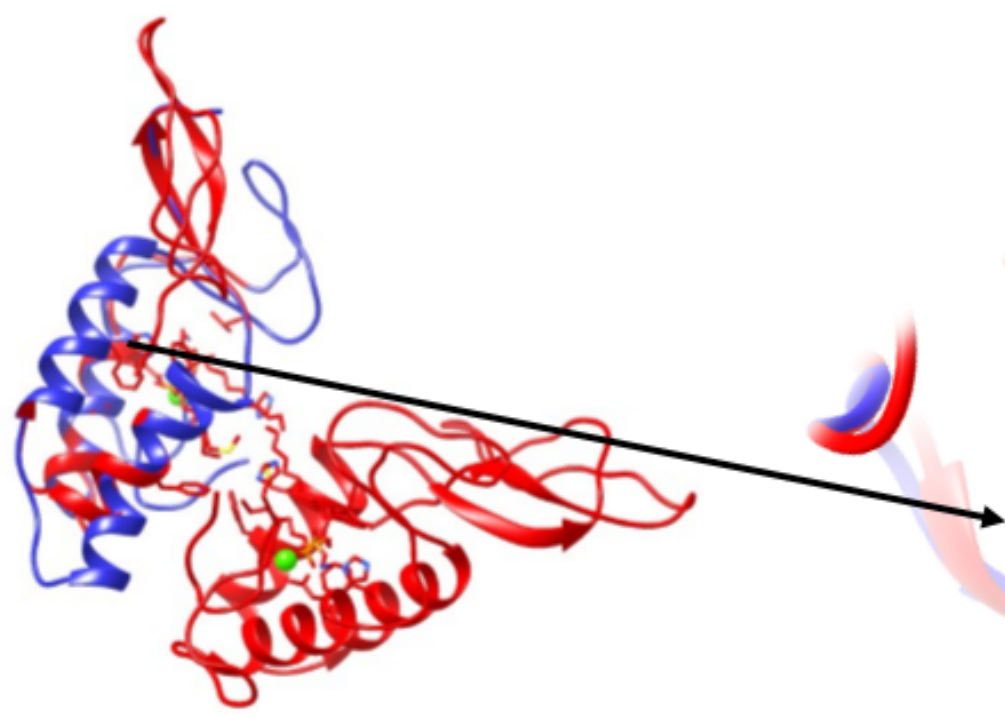

(C)

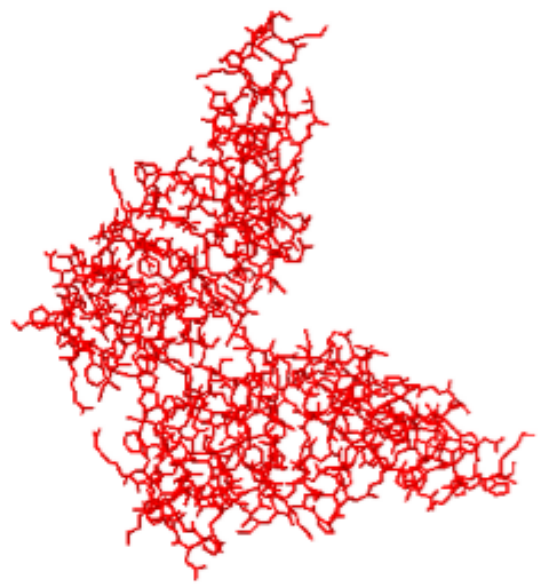

(B)

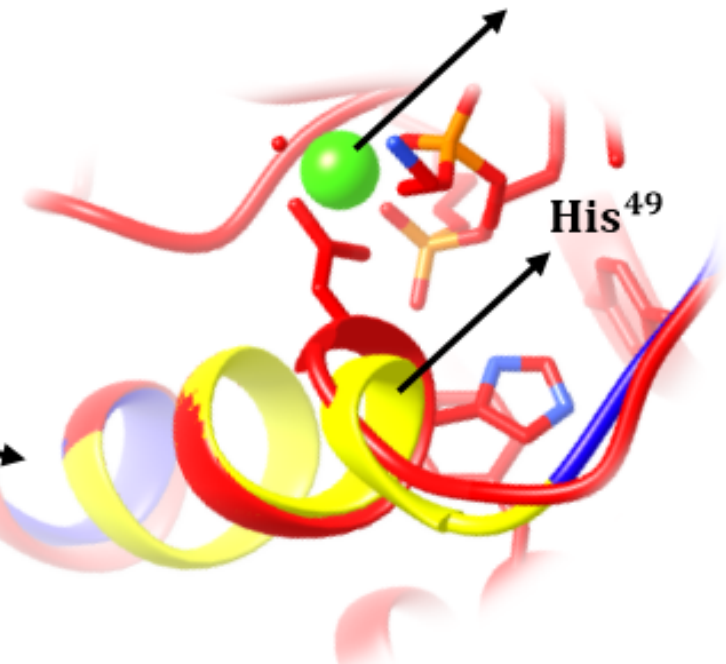

(D)

\section{Figure 4}

Superimposition of 3D structure of bee venom and S. maurus PLA2. 3D structure of S. maurus PLA2 is similar to the chain A of bee venom PLA2. (A) and (B): the 3D structure predicted by DeepView/Swisspdbviewer (version 4.10) software. (C): The Superimposition predicted by UCSF Chimera (version 1.14) software. In both pictures, the blue color shows S. maurus PLA2 and the red color shows bee venom PLA2. (D): The yellow color shows S.maurus active site. 


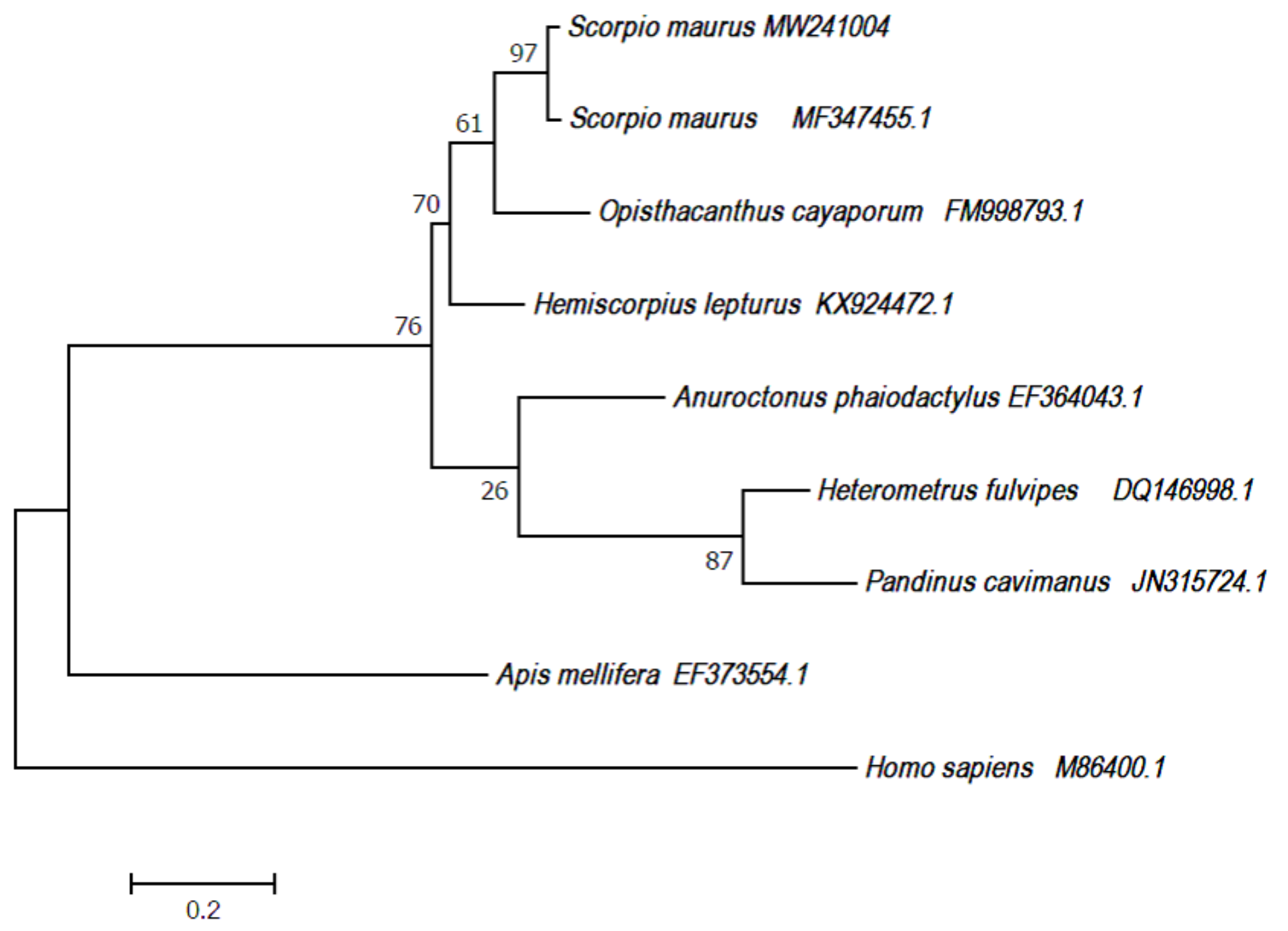

Figure 5

Phylogenetic relationship between different species of scorpion, Apis mellifera, and Homo sapiens based on PLA2 sequences. The accession number of each sequence has been shown in front of its name. The percentage of replicate trees in which the related taxa clustered together in the bootstrap test (1000 replicates) has been displayed next to the branches. The scale bar corresponds to 0.2 substitutions per nucleotide 


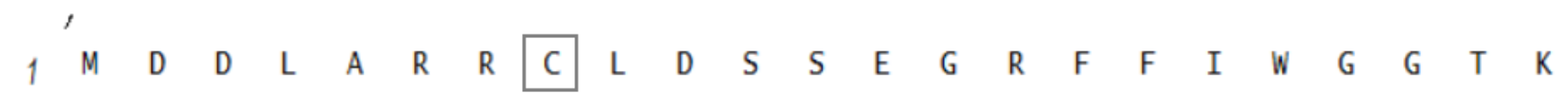

1 ATGGATGATTTGGCAAGAAGATGCTTAGATTCATCAGAgGGAAGATTTTTTATATGGGGAGgGACCAAGT 24 W $\mathrm{C}$ C G 71 GGTGTGGGCCAGGCAACGAGGCGGAAAATGAATCTGATCTTGGTCCTCTTGAAGCTGATAAGTGTTGCCG 4t

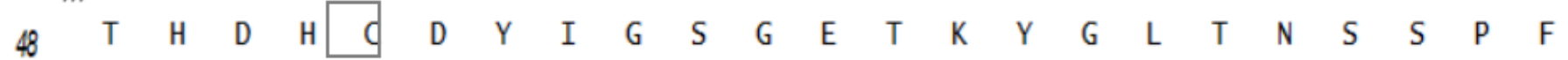
141 CACTCATGACCACTGCGACTATATAGGGTCTGGAGAAACGAAATATGGACTAACTAACAGCTCTCCT T TC $2 t$

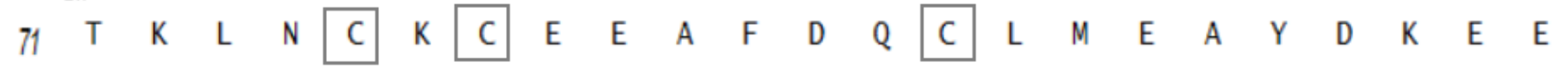

211 ACTAAGTTGAACTGCAAATGCGAAGAAGCTTTCGATCAGTGCTTGATGGAAGCTTATGACAAAGAGGAGG 287

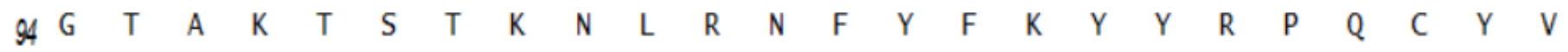
281 GCACTGCAAAGACATCGACTAAAAAT T TGCGAAACTTT TATT TCAAATATTATAGACCACAGTGCTATGT

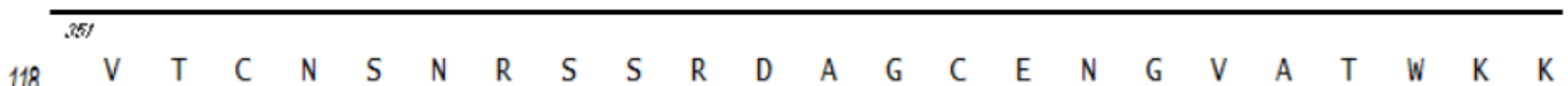
351 TGTGACATGTAACAGTAATAGG TCGAGCAGGGATGCAGGATGTGAAAATGGAGT TGCTACCTGGAAAAAG 427

141 S $\quad Y \quad K \quad D$

421 AGCTACAAAGAC

\section{Figure 6}

Nucleotides and predicted amino acid sequence of S. maurus PLA2. Nucleotide and amino acid numbers appeared on the left. The middle-letter at above the nucleotide sequence is coded the amino acid sequence. Coding sequence and predicted amino acid sequence. Coding sequence and predicted amino acid sequence of PLA2. The disulphide bonds between cysteine residues in the protein structure have been signed by black boxes. The conserved domines of target PLA2 amino acid length with PLA2 superfamily have been shown by black line. 